\title{
Enhancing the Data Collection Rate of Tree-Based Aggregation in Wireless Sensor Networks
}

\author{
Özlem Durmaz Incel \\ Department of Computer Science \\ University of Twente \\ Enschede, 7522NB, the Netherlands \\ Email: o.durmaz@cs.utwente.nl
}

\author{
Bhaskar Krishnamachari \\ Department of Electrical Engineering - Systems \\ University of Southern California \\ Los Angeles, CA 90089, USA \\ Email: bkrishna@usc.edu
}

\begin{abstract}
What is the fastest rate at which we can collect a stream of aggregated data from a set of wireless sensors organized as a tree? We explore a hierarchy of techniques using realistic simulation models to address this question. We begin by considering TDMA scheduling on a single channel, reducing the original problem to minimizing the number of time slots needed to schedule each link of the aggregation tree. The second technique is to combine the scheduling with transmission power control to reduce the effects of interference. To better cope with interference, we then study the impact of utilizing multiple frequency channels by introducing a simple receiver-based frequency and time scheduling approach. We find that for networks of about a hundred nodes, the use of multi-frequency scheduling can suffice to eliminate most of the interference. The data collection rate then becomes limited not by interference, but by the maximum degree of the routing tree. Therefore we consider finally how the data collection rate can be further enhanced by the use of degree-constrained routing trees. Considering deployments at different densities, we show that these enhancements can improve the streaming aggregated data collection by as much as 10 times compared to the baseline of single-channel data collection over non-degree constrained routing trees. Addition to our primary conclusion, in the frequency scheduling domain we evaluate the impact of different interference models on the scheduling performance and give topology-specific bounds on time slot and frequency channel requirements.
\end{abstract}

\section{INTRODUCTION}

Periodic collection of aggregated data from sensors to a common sink over a tree topology is a fundamental operation in wireless sensor networks (WSN). In many such applications, it is of interest to maximize the rate at which the sink can receive aggregated data from the network [1]. For instance, it has been noted that in networked structural health monitoring more than 500 samples per second are required to efficiently detect damages [2].

Time division multiple access (TDMA) scheduling is a natural solution for such periodic data collection applications [3], [4]. Consider a repeated frame of $k$ time slots in which each link of the data gathering tree is scheduled once. In steady state (once a pipeline is established), the sink will receive aggregated information from all nodes in the network once per frame, i.e. once every $k$ slots. In this framework, maximizing the data collection rate corresponds exactly to minimizing the frame length. This is the focus of our work ${ }^{1}$.

We explore a number of techniques in order to address the basic question: "how fast can aggregated data be streamed to the sink"? These techniques provide a hierarchy of successive improvements. The simplest approach is to do some form of interference-aware minimum frame-length TDMA-scheduling that enables spatial reuse. The second step is to combine the scheduling with transmission power control. The third step is to consider the use of multiple frequency channels. We show that once multiple frequencies are employed along with spatial-reuse TDMA, the aggregated data collection rate often becomes no longer interference-limited, but rather topologylimited. Thus, the final step to enhance the rate of periodic aggregated data collection is to use an appropriate degreeconstrained tree topology. Our primary conclusion is that combining these techniques can provide an order of magnitude improvement in the rate compared to the simple approach of TDMA scheduling on a single channel with minimum-hop routing trees.

We evaluate different design choices using simulations that use realistic channel models and radio parameters typical of WSN radio devices. The following are some of the key contributions and findings of this work:

- Evaluation of power control in a realistic setting: Moscibroda [1] has shown in a recent theoretical study that under idealized settings (unlimited power, continuous range) power control mechanisms can provide unbounded improvements in the asymptotic capacity of aggregated data collection. We employ the optimal power control algorithm proposed by El Batt and Ephremides [5] in a practical setting taking into account the limited discrete power levels available in today's radios. We find that for moderate size networks of 100 nodes, power control can reduce the number of time slots by $15-20$ percent.

- Comparison of interference models: In the literature, there are two common approaches to model interference. The

\footnotetext{
${ }^{1}$ While we do not focus on minimizing energy usage directly, we note that the formulation we explore provides energy efficiency in two ways: aggregation reduces the total data transmitted, and the TDMA scheduling can eliminate collisions in addition to permitting nodes to go into sleep mode during inactive slots. Furthermore, for applications with a fixed data rate, minimizing the schedule length allows for a longer sleep period in each cycle.
} 
protocol model is a graph theoretic approach with the assumption that a message is correctly received if no other sender transmits at the same time in close proximity. The advantage of this approach is that it enables the use of simple graph-coloring based scheduling algorithms. On the other hand, the protocol model may fail in practice since interference is not a binary phenomenon [6]. The model can also be pessimistic in the sense that two nearby communications can simultaneously take place if the interference level is tolerable. A richer model that can capture the interference from multiple senders is the physical model also named as SINR (signal to interference and noise ratio) model. We evaluate the impact of both models on the scheduling performance. We find that the use of the graph based model fails most in sparse network deployments with higher path loss exponents, but even then only about 12 percent of nodes are scheduled incorrectly. This suggests that graph-based scheduling design followed by SINR-based validation and repair maybe an acceptable compromise in practice.

- Receiver-based frequency-time scheduling: In order to use multiple frequency channels, we introduce a simple receiver-based frequency-time scheduling mechanism suitable for aggregation trees in WSN. The basic idea of our scheme is to associate frequencies with receivers rather than transmitters, and to allocate frequencies greedily (with reuse) to minimize interference.

- Bounds on time and frequency requirements: We provide topology-specific bounds on the schedule length and frequency channel requirements. These bounds can be quite useful in characterizing fundamental performance since they can be determined readily from in-network interference measurements for a given routing topology.

- Impact of adjacent channel interference: One simplifying assumption often made about frequency channels is that the transmissions on different frequencies are orthogonal, or in other words interference-free. However, assumption of perfect orthogonal channels may fail in practice because of interference from adjacent channels. We find that the practical impact of this simplifying assumption depends upon the particular radio platform employed, as well as the density of deployment.

- Identification of connectivity bottleneck: On a tree topology whenever there are multiple senders (children) assigned to the same receiver (parent), each of these senders have to be allocated a different time slot since a single half-duplex transceiver can receive from only one node at a time. When the interference limitation can be eliminated or mitigated to a large extent using multiple channels and power control, we find that the maximum degree on the tree becomes the bottleneck for scheduling performance, especially in denser deployments.

- Routing enhancements: We investigate a degree constrained routing tree construction mechanism, which turns out to be more useful. Simulation results show that combining a simple degree constrained tree construc- tion algorithm with frequency scheduling can reduce the schedule length up to 10 times than scheduling on simple minimum-hop routing trees on a single frequency channel.

The remainder of the paper is organized as follows: in Section II we explain the mechanisms that we use to investigate the scheduling performance. Section III discusses different design possibilities on modeling co-channel and adjacent channel interference. Section IV presents the possible upper and lower bounds on the time and frequency requirements. Section V gives the detailed simulation based evaluation of the discussed methods. Section VI summarizes some of the related work. Finally, Section VII provides the concluding remarks.

\section{MECHANISMS}

\section{A. Preliminaries}

Before explaining the studied mechanisms, we first express the preliminary design details and assumptions:

- We consider a static wireless sensor network. The sensor nodes periodically sense the environment and send their readings over a multi-hop tree topology.

- Time is divided into equal sized slots that are grouped into frames. We focus on minimizing the length of the frame such that each node is assigned one time slot.

- We consider minimum-hop routing trees where all the nodes select a parent node where they transmit their readings to be forwarded towards the sink node.

- We assume all the nodes in the network are sources and the data is aggregated such that the data coming from different sources are combined into a packet(s) before forwarding. If the incoming packets cannot be combined in a single packet and multiple packets have to be forwarded, we assume each time slot is long enough to transmit those packets. This is a reasonable assumption since the size of the sensor readings is usually very small.

Figure 1 shows the relationship between the schedule length and the aggregated data rate. The numbers on the links show the assigned time slots and the numbers inside the circles represent the node id's. On the left of the figure we see the schedule showing the received packets from the associated senders by each parent on each time slot. After frame 1, once the sink gets initial data from each source (a pipeline is established), the same schedule is repeated and the sink collects the aggregated data from the network at a rate of 3 time slots. Thus, the schedule length should be minimized to improve the data collection rate.
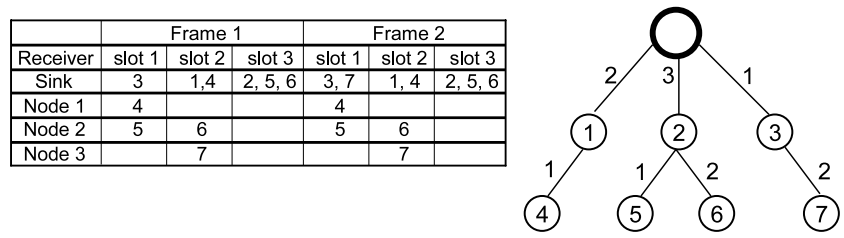

Fig. 1. Relationship between data collection rate and schedule length 


\section{B. Joint Scheduling and Power Control}

El Batt et al. [5] introduced a cross layer method for joint scheduling and power control in wireless multi-hop networks. They proposed an optimal distributed algorithm to improve the throughput capacity of wireless networks. The aim is to find a TDMA schedule which can support as many transmissions as possible in each time slot. We use their algorithm to investigate the impact of power control on the scheduling performance.

The solution is composed of 2 phases: scheduling and power control. It is to be executed at the beginning of each time slot in order to cope with excessive interference levels. The scheduling phase searches for a transmission schedule which is defined to be valid if no node is to transmit and receive simultaneously and no node is to receive from more than one neighbor at the same time. Power control phase iteratively searches for an admissible schedule which means that a set of transmission powers is available to satisfy the SINR (signal to interference and noise ratio) constraints for all links in the given valid schedule. In each iteration nodes adjust their transmission powers as follows:

$$
P_{\text {new }}=\frac{\beta}{S I N R} * P_{\text {current }}
$$

where $P_{\text {new }}$ is the new transmission power level in the next iteration, $P_{\text {current }}$ is the current transmission power level and $\beta$ is the SINR threshold.

If the maximum number of iterations is reached and still there are nodes which cannot meet the SINR constraints, i.e., if the valid scenario is not admissible, the scheduling algorithm excludes the link with the minimum SINR. The power control algorithm is repeated until an admissible transmission scenario is found. Then, the nodes start transmission using the computed transmission powers in the current slot.

\section{Frequency and Time Scheduling}

The use of multiple frequency channels is an efficient way to improve the capacity of wireless networks. Simultaneous transmissions on different frequencies (if the frequencies are not orthogonal, different frequencies may also be conflicting. We use non-conflicting frequencies and different frequencies interchangeably in the text) can take place without interference in the same spatial neighborhood.

In this section we introduce a simple scheduling method which separately assigns the time slots and frequencies on a tree topology. Motivation for this proposal is as follows:

- Intersecting links, which are defined as the links with a common destination (Figure 2), cannot transmit on the same time slot since they have to wait for each other's transmission. Assigning non-conflicting frequencies to these nodes does not improve the situation, either. Then the receiver should be assigned a frequency and the senders should use this frequency to transmit to the parent.

- Interfering links are the links which create/face interference if they are scheduled simultaneously. Figure 2 shows an example where the dotted line represents interference.
Interfering links should not get the same time slot and frequency. Since our aim is to minimize the number of time slots, the best option then is to assign the same time slot on non-conflicting frequencies.

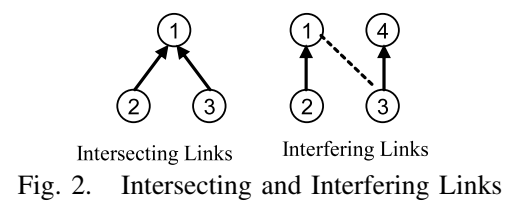

The method is composed of 2 phases: frequency scheduling and time slot scheduling. In the frequency scheduling phase, the receivers, i.e. the parents on the tree, are assigned frequencies. The goal of the frequency assignment phase is to schedule the interfering links on non-conflicting frequencies such that the receptions at the parents of the interfering senders are not disturbed. Initially, all the nodes operate on the same frequency. The method collects information about the interfering links (we consider both graph based and SINR based interference models and present the results in Section V). According to the collected information, at each step the most interfered parent (the parent with the highest number of interfering links) is assigned a frequency, if one is available. If not, the parent node and the associated children remain on the initial frequency and the interference conflicts have to be solved in the time slot assignment phase.

After the frequency scheduling, the algorithm continues with the time slot assignment to the senders. Similar to the power control approach, a node can be scheduled such that it cannot transmit and receive simultaneously and cannot receive from more than one neighbor at the same time, due to the transceiver limitations. If the parents of all interfering senders could be assigned different frequencies (this means the interference is totally eliminated), we can skip the SINR check. If not, during the time slot assignment, the SINR condition is checked among the interfering senders.

Figure 3 shows a scheduling example on a tree topology. In the first part of the figure, the solid lines between the nodes show the transmission links whereas the dotted lines show the interfering links. The numbers inside the circles represent the node id's. The second part of the figure shows the tree after time slot assignment with a single frequency channel. The numbers on the links show the assigned time slots. In this case, it takes 6 time slots to schedule the network. In the third part of the figure we see how the scheduling is performed with 2 frequencies. First, the frequencies are assigned to the parents (represented inside the boxes next to each parent, $F 1$ is the initial frequency). Then, the time slots are assigned to the senders. With 2 frequencies, the network is scheduled in 4 slots. The last part shows the case with 3 frequencies. The network is then scheduled in 3 time slots. We achieve a $\% 50$ reduction on the schedule length thus the data collection rate at the sink node is doubled with the sufficient number of frequencies.

The receiver based frequency assignment makes the algorithm suitable for tree topologies and avoids the overhead of fre- 


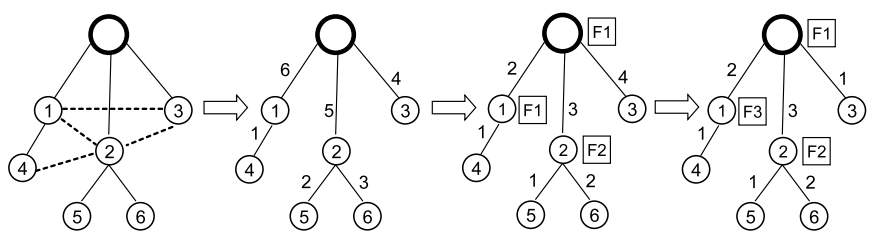

Fig. 3. Frequency and Time Scheduling on a Minimum-Hop Routing Tree

quent frequency switching for the transceiver since the nodes switch at most between 2 frequencies. It may be argued that, an interfering link does not always disturb all the children of a parent node. Thus, assigning a frequency to a parent limits the communication possibilities for those children. To investigate this issue, by extensive simulations, we compared the performance of our algorithm with a different approach where each sender is assigned a time slot and a frequency. The performance is observed to be similar, however we cannot present the results in this paper due to the space limitations.

\section{Routing Strategies, Parent Selection}

In the previous sections we have discussed the methods to cope with interference. Other than interference, connectivity may also limit the performance of scheduling. Consider the nodes that select the same parent. They have to wait for each others' transmission which simply increases the length of a schedule. In this section we investigate the methods that can adjust the degree of connectivity on a tree topology.

One option would be to construct balanced trees. We compared the scheduling performance on minimum-hop balanced and unbalanced trees. However, no improvement is observed with balanced trees since the sink node often remains the high-degree bottleneck (due to the space limitations we cannot represent the results in this paper). To avoid the bottlenecks, there should be a limitation on the number of children per parent. Thus, we explore scheduling on degree constrained tree topologies.

A degree-constrained minimum-hop tree is constructed using a modified version of Dijkstra's shortest path algorithm. Consider a graph $G(V, E)$ and a given degree constraint max_degree. Each node $n$ keeps a value for the number of its children $C(n)$ with an initial value $=0$ and hop count to the sink $H C(n)$ with an initial value $=\infty$. The algorithm starts with a set $T$ that contains the sink node $s(H C(s)=0)$, at each iteration we add a node $m \notin T$ to $T$ with the following constraints:

- there is a node $m^{\prime} \in T$ such that edge $\left(m, m^{\prime}\right) \in E$,

- $C\left(m^{\prime}\right)<$ max_degree - 1 ,

- the hop count to the sink $=H C(m)$ is minimized.

The updates are made as $H C(m)=H C\left(m^{\prime}\right)+1$ and $C\left(m^{\prime}\right)=C\left(m^{\prime}\right)+1$. The algorithm stops when $|T|=|V|$ or when no more nodes can be added since the degree of the all nodes in $T$ have reached the max_degree.

To clarify the gains with this method, consider the case when all $n$ nodes are in range of each other and the sink. If the nodes select their parents according the minimum hop criteria without a degree constraint, all the nodes will select the sink as a parent and this schedule will take $n$ time slots. On the other

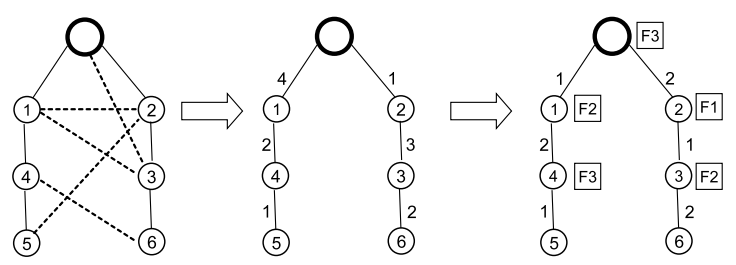

Fig. 4. Scheduling on a Degree Constrained Tree

hand, if we limit the number of connections per node as 2 , this will result in 2 subtrees rooted at the sink. If there are enough number of frequencies to eliminate all the interference then the network can be scheduled in 2 time slots and we achieve a factor of $n / 2$ reduction in the schedule length. Figure 4 shows the same network as in Figure 3 with a different routing tree where the degree of a node is constrained to 2 . The second part of the figure shows the time slot scheduling which takes 4 time slots on a single channel frequency. The last part shows when time slots are scheduled over different frequency channels. This takes 2 time slots to schedule all the links which is "3" times better than the baseline with a single frequency over a non-degree constrained tree, given in Figure 3.

\section{MOdels FOR DESIGN}

\section{A. Interference Models}

As we discussed in the introduction, there are two different interference models that are commonly used in the literature: protocol model and the physical model. According to the protocol model the transmission from a node $i$ to a node $j$ is successful, if for every other node $k$ simultaneously transmitting, the following condition holds

$$
d(k, j) \geq(1+\Delta) d(i, j),(\Delta>0)
$$

where $d(i, j)$ is the distance between nodes $i$ and $j$, and $\Delta$ is a guard parameter that ensures that concurrently transmitting nodes are sufficiently further away from the receiver to prevent excessive interference.

On the other hand, physical model is an SINR based model that takes into account multiple transmissions. Given a transmission from node $i$ to node $j$, the SINR value at receiver $j$ is computed as follows:

$$
S I N R_{i j}=\frac{\alpha_{i j} P_{i}}{N_{j}+\sum_{k \neq i} \alpha_{k j} P_{k}}
$$

where $\alpha$ is the path loss factor, $P_{i}$ and $P_{k}$ are the transmission powers for $i$ and $k$, and $N_{j}$ is the amount of ambient noise experienced by receiver $j$. Transmission is successful if the corresponding SINR at receiver $j$ is above the threshold value:

$$
S I N R_{i j} \geq \beta
$$

where $\beta$ is the SINR threshold. In Section V-B.1, we investigate the correctness of the graph based model and the effects of the both models on scheduling performance. 


\section{B. Orthogonal Frequencies vs. Interfering Frequencies}

In the current literature on multi-channel protocols, mostly either it is assumed that the channels are perfectly orthogonal (interference-free) or the use of overlapping channels is simply avoided. Assumption of perfect orthogonal channels fails in practice since radio signals are not bound to a single point in the spectrum but are distributed around a mid frequency so that channel overlap/interference is observed between adjacent bands. On the other hand, the use of only orthogonal channels cannot utilize the spectrum efficiently. For instance, the $802.11 \mathrm{~b}$ standards define 11 channels of which only three are orthogonal. Careful use of not only 3 channels but all 11 channels by controlling the interference can significantly improve the system performance [7].

Interference between overlapping channels is influenced by the transmission power, distance between transmitters, channel spacing and transceiver characteristics [8]. In Section V, we compare the impact of orthogonal frequencies and interfering frequencies on the scheduling performance for two different transceiver platforms. Moreover, we investigate the correctness of schedules generated with the orthogonal frequencies assumption.

\section{Performance Bounds}

In this section we present the theoretical upper and lower bounds on time slot and frequency requirements that are based on max-degree bounds for graph coloring [9].

\section{A. Bounds on the time slots}

In this section, we present the bounds on the required number of time slots to schedule all the links in the network. We use the following formulation and notation:

Given a tree $T$, and the interference graph I of $T$, schedule all links of $T$ with minimum number of time slots.

The graph $T$ includes the communication links $(c, p)$ between the children and the associated parents. The interference graph $I$ is a graph which includes the links $(i, p)$ that cause the SINR to be less than the threshold $\beta$ for a link between parent $p$ and any child of $p$. We define the interfering parents as a pair of parents $\left(p, p^{\prime}\right)$ such that the simultaneous transmission of any child $c^{\prime}$ of $p^{\prime}$ interferes with a transmission of any child $c$ of $p$.

After frequency assignment, if all the interference is eliminated such that each interfering parent has a disjoint frequency, then the lower bound on the required number of time slots is,

$$
\max \left(\Delta_{1}+1\right)(\text { in } T)
$$

where $\Delta_{1}$ is the one-hop degree of a node, i.e., the number of one-hop neighbors of any of the nodes on graph $T$.

If the interfering parents could not be allocated different frequencies, then the nodes within their 2-hop interference neighborhood should be assigned different time slots. Let $I^{\prime}$ represent the new interference graph after frequency assignment. The upper bound is

$$
\max \left(\Delta_{2}+1\right)\left(\text { in } I^{\prime}\right)
$$

where $\Delta_{2}$ is the 2-hop degree of a node on $I^{\prime}$, i.e., the number of two-hop neighbors of any node on graph $I^{\prime}$.

We compare the performance of the algorithm with the calculated bounds in Section V-C.1.

\section{B. Bounds on the frequencies}

The receiver-based frequency and time scheduling approach aims to assign different frequencies to all interfering parents. Upper bound is the number of all parents that do interfere with a given parent. Let set $P$ include the set of all parents and $P(p)$ represent the interfering parents with parent $p \in P$. Then the upper bound is:

$$
\max (|P(p)|)(p \in P)
$$

If a parent $p^{\prime} \in P(p)$ does not interfere with another parent $p " \in P(p)$, then they can be assigned the same frequency. Let $P^{\prime}(p) \subset P(p)$ include the parents that cannot be assigned the same frequency and one parent from the parents that can be assigned the same frequency. Then the lower bound is:

$$
\max \left(\left|P^{\prime}(p)\right|\right)(p \in P)
$$

We present the comparisons of actual performance with the calculated bounds in Section V-C.2.

\section{EVALUATION}

We use a simulation based approach using Matlab to evaluate the impact of different mechanisms on the scheduling performance. Nodes are randomly deployed over the area. Terrain dimensions are varied between $25 \times 25$ and $150 \times 150 \mathrm{~m}^{2}$ (when $L>150$, the network becomes disconnected/partitioned) to simulate different levels of density whereas the number of nodes is kept as 100. During the topology construction phase the node with id "1" is always selected as the sink node. For different parameter settings, we repeat the simulations at least 1000 times.

We use an exponential path loss model for signal propagation, with different path loss exponents, $\alpha$, varying between 3 and 4 . We simulate the behavior of the CC2420 radio [10] which is used on the Telosb and Tmote sensor mote platforms. The transmission power can be adjusted between $-24 \mathrm{dBm}$ and OdBm over 8 different levels. SINR threshold is $\beta=3 d B$ and the transceiver is capable of operating on 16 different frequency channels with a channel spacing of $5 \mathrm{MHz}$ within the $2.4 \mathrm{GHz}$ band.

\section{A. Impact of Power Control}

In this section we evaluate the impact of transmission power control on the scheduling performance. We investigate two cases: nodes transmit with the maximum transmission power and nodes adjust their transmission power according to the power control algorithm which is explained in Section IIB. The results are shown in Figure 5. The x-axis shows the density of the deployment which is defined as: 


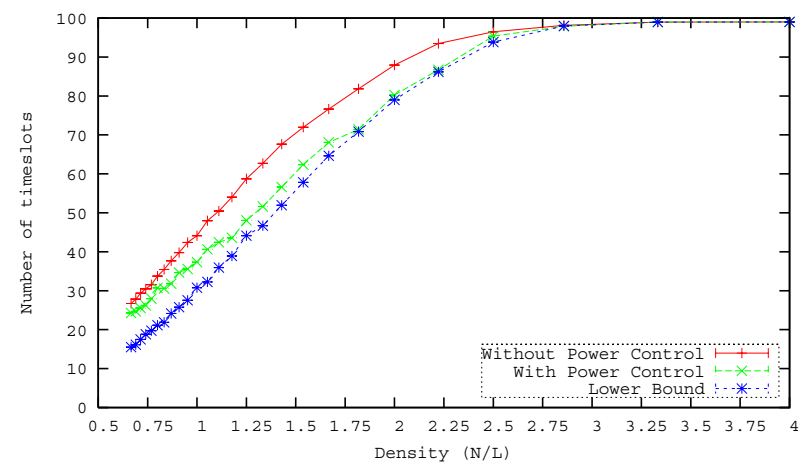

Fig. 5. Scheduling With and Without Transmission Power Control

$$
d=\frac{N}{L}
$$

where $N$ is the number of nodes and $L$ is the length of the area. The y-axis shows the number of time slots required to schedule all the nodes in the network.

The required number of time slots increases with the density. As the density increases, both the levels of interference and connectivity increase and consequently the possibility of concurrent transmissions decreases. In the worst case $(d=4)$, all the nodes are in the transmission range of each other. Since we employ a minimum-hop routing scheme, all the nodes select the sink node as the destination. In this case the network can be scheduled with 99 time slots (since sink does not transmit), whether the nodes adjust their transmission power or not. In sparser scenarios, if the nodes employ a power control mechanism then the network can be scheduled in less time since the level of interference is reduced. We achieve a factor of $10-20 \%$ reduction in the number of time slots in the best case.

As theoretically proved in [1], power control can help in reducing the effects of interference on scheduling. However, in practice we cannot achieve the lower bound, which is the maximum degree in the network, in all cases since we cannot eliminate interference totally. This is due to the discrete power levels and the limited range of the transmission power. For instance $\mathrm{CC} 2420$ radio has 8 different levels between 0 $\mathrm{dBm}$ and $-24 \mathrm{dBm}$. Moreover, there is the sensitivity level of $-95 \mathrm{dBm}$ for the transceiver to be able to decode a signal (sensitivity is the minimum received power that results in a satisfactory bit error rate). This also limits the sender to further reduce the transmission power.

\section{B. Impact of Frequency and Time Scheduling}

In this section we analyze the performance of receiver based frequency and time scheduling method which is introduced in Section II-C. Figure 6 shows the results when the network is scheduled with the SINR interference model. The X-axis shows the density whereas the $y$-axis shows the required number of time slots. Different lines show different numbers of available frequency channels.

The network can be scheduled in less number of time slots compared with the transmission power control mechanism. In sparser deployments $(d<1)$, we achieve a reduction of

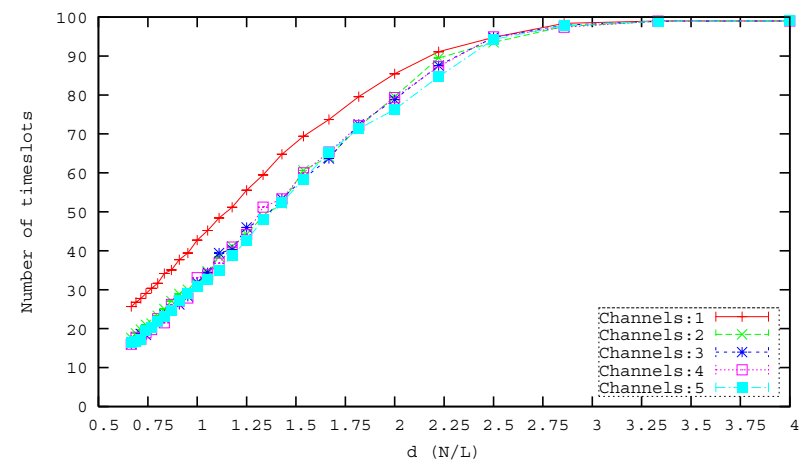

Fig. 6. Frequency and Time Scheduling with SINR based Interference Model

$40 \%$ on the schedule length. When the network gets denser, having more frequencies does not help due to the increased connectivity in the network. In the worst case the problem turns into a single-hop scheduling since sink is the only destination for all nodes thus the degree of the sink is the dominant limiting factor. Another interesting observation is that when we have two or more different frequencies, the scheduling performance does not change since the interference limitation is already eliminated with two frequencies. Beyond this point connectivity constraint limits the performance.

Figure 7 shows the results for the same setting with the graph based interference model instead of the SINR model. The results are observed to be similar. However, when there is a single frequency channel available, the number of required time slots is higher than that with the SINR model (Figure 6). As we discussed in Section I, graph based interference models may over/under estimate the level of interference.

1) Comparison of Graph Based and SINR Based Interference Models: As we discussed, graph based interference models may not be realistic to capture interference with multiple senders or may overestimate the interference. In this section, we present the failure rate of graph based interference model: a generated schedule is examined to be feasible or not according to the SINR constraints. Figure 8 shows the error rate in terms of the number of nodes that are not correctly scheduled out of 100 nodes versus density. Different lines on the figure show different number of frequencies and different values of path loss exponents $(3,3.5,4)$. Error rate is much higher in sparser deployments than in denser deployments. In sparser deployments, interference created by individual transmitters is

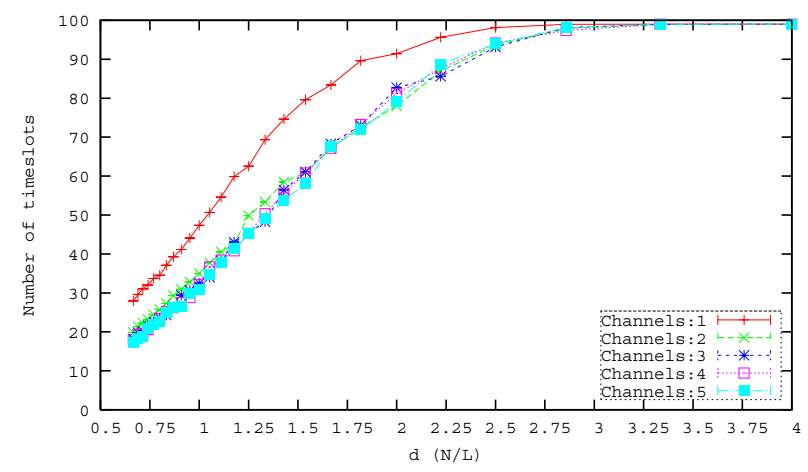

Fig. 7. Frequency and Time Scheduling with Graph based Interference Model 


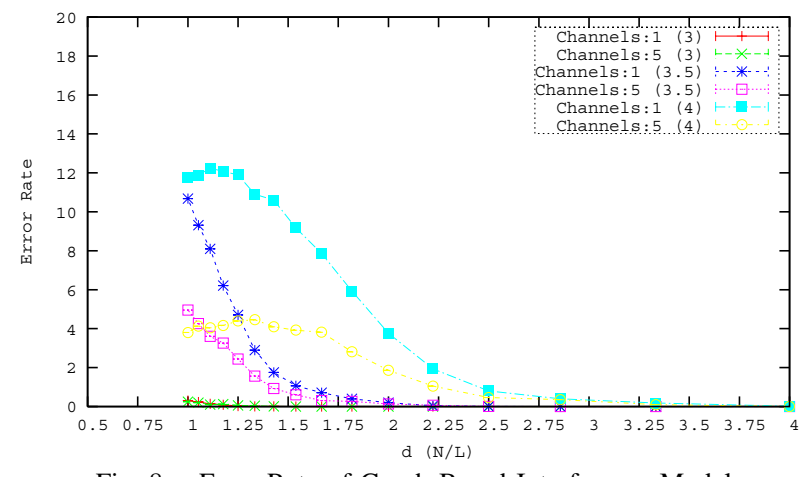

Fig. 8. Error Rate of Graph Based Interference Model

not high enough to jam the parallel transmissions. However, the cumulative interference from multiple senders can be detrimental which cannot be captured by a graph based interference model. On the other hand, in denser deployments an individual transmitter can also jam a simultaneous transmission since the distances between the nodes are smaller and the level of interference is higher.

Path loss parameter is an important factor on the received signal strength and also on the level of interference. When the path loss exponent is set to a smaller value such as 3 , received signal strength is high and also the level of interference by an individual jammer is high. If the path loss exponent is high, individual transmitters may not jam the desired transmission but multiple senders may. Having less number of frequency channels also increases the error rate because more transmissions occur on the same channel.

2) Impact of Frequency Orthogonality: As we discussed in Section III-B, the assumption of perfect orthogonal frequency channels may fail in practice. The adjacent/alternate channel rejection values of the transceiver are given as follows: $+5 \mathrm{MHz}:-45 \mathrm{~dB},-5 \mathrm{MHz}:-30 \mathrm{~dB},+10 \mathrm{MHz}:-54 \mathrm{~dB}$, $-10 \mathrm{MHz}:-53 \mathrm{~dB}, \geq+15 \mathrm{MHz}:-62 \mathrm{~dB}, \leq-15 \mathrm{MHz}:-62 \mathrm{~dB}$. These values indicate how much power the receiver can tolerate on a nearby frequency/channel, and still can receive on a desired channel.

We have investigated the scheduling performance with the overlapping frequencies. Due to the space limitations we do not present the results. Results are observed to be similar with the orthogonal frequency results, shown in Figure 6 since CC2420 radio has high adjacent/alternate channel rejection values such that the channels behave like orthogonal.

Although we do not observe any performance degradation with overlapping frequencies, a schedule generated by the orthogonality assumption may not be correct. We do not present the results with the CC2420 radio since the error rates are small and can be avoided. Figure 9 shows the error rates with another transceiver platform: Nordic nRf905 radio [11]. nRf905 radio has smaller channel distance $(200 \mathrm{kHz})$ between the consecutive frequencies and smaller adjacent/alternate channel rejection values compared with the CC2420 radio. The $\mathrm{x}$-axis shows the density and the $\mathrm{y}$-axis shows the error rate, i.e. the number nodes that are scheduled incorrectly out of 100 nodes. In sparser scenarios, initially the error rate is small.

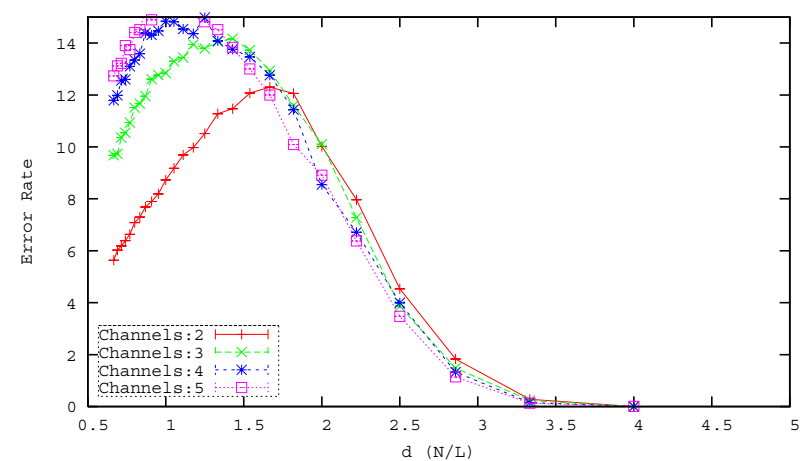

Fig. 9. Error Rate of Orthogonal Frequencies Assumption (nRf905 radio)

Then it starts increasing since the network gets denser and the interference level increases. When the interference level is higher some nodes select the next available channel according to the orthogonal channel assumption. However, the level of interference experienced on the next channel can still be high to disturb the transmission. After the peak point, the number of nodes that can be simultaneously scheduled is decreasing and the connectivity constraint becomes the dominant factor instead of the interference constraint.

\section{Comparisons with the Analytical Bounds}

1) Bounds on Time Slots: In this section we compare the simulation results with the analytical bounds that we present in Section IV. We show an example setting in Figure 10 where the number of nodes is 100 and the terrain dimensions are $100 \times 100 \mathrm{~m}^{2}$. The $\mathrm{x}$-axis shows the number of available frequencies, and the $y$-axis shows the number of required time slots to schedule the network. When all the nodes operate on the same frequency channel, number of time slots is much higher than the optimal case, i.e. the lower bound. This is due to the interference. When the number of frequencies is increased to 2, the interference is eliminated and the dominating constraint is the connectivity.

We also investigate the bottleneck in terms of the connectivity constraint. We simulated the scheduling behavior on unbalanced and balanced trees. However, no difference is observed since the sink node becomes the high-degree bottleneck on minimum-hop balanced trees. Since the maximum degree is similar in both cases, the bounds on time slots are observed to be the same.

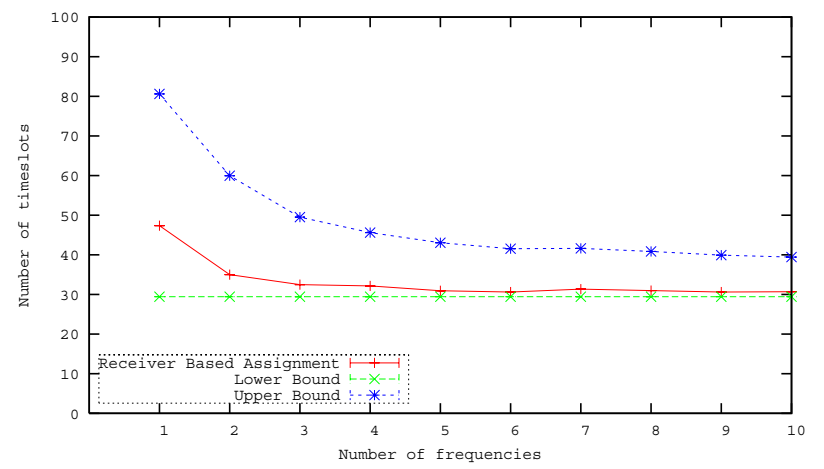

Fig. 10. Bounds on the number of time slots (100 nodes, $100 * 100 \mathrm{~m}^{2}$ ) 


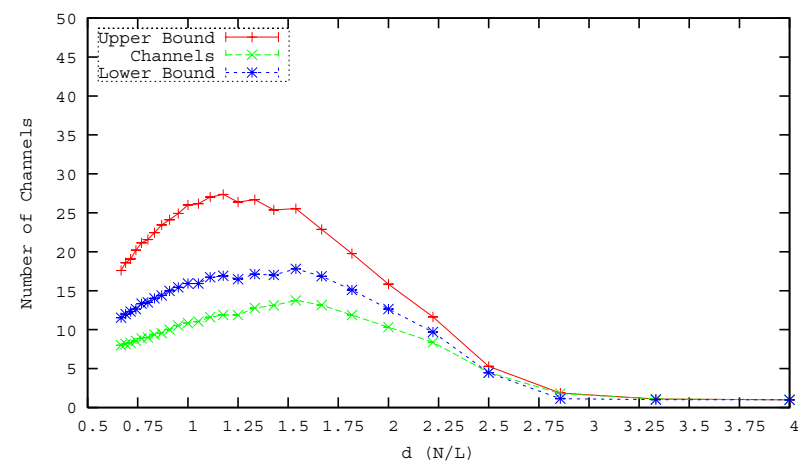

Fig. 11. Bounds on the number of frequencies

2) Bounds on the number of frequencies: Receiver based time slot and frequency scheduling aims to schedule all the interfering parents on different frequencies. We investigate how the required number of frequencies change with density. Figure 11 shows the bounds on the number of frequencies. The number of required frequencies initially increases since the interference increases with the density. After the peak point although the network gets denser, the number of nodes selected as a parent is decreasing therefore the required number of unique frequencies decreases. In the densest setting, since the sink node is the only parent, the number of frequencies is 1 .

\section{Impact of Parent Selection}

In the preceding sections, we have discussed the performance of the methods to eliminate interference. We have assumed that the nodes select their parents according to the minimum hop criteria during tree construction. Accordingly, we observe that we can easily overcome interference limitation with multiple frequencies but connectivity limits the performance. In this section we investigate the scheduling performance on degreeconstrained trees. Degree constrained tree construction problem with the minimum hop constraint is NP-Complete [12] which can be shown by a reduction to the Hamiltonian path problem. For simplicity, we assume well-connected graphs.

Figure 12 shows the required number of time slots to schedule the network versus the degree of the tree topology. The nodes are scheduled with the receiver based frequency and time scheduling method and 16 frequency channels are available. Different lines on the figure show different density values. In the densest setting when $d=4$, the required number of time slots hits the lower bound at degree 8 . In smaller degrees, the number of frequencies is not sufficient to overcome interference but the required number of time slots at degree 2 , for instance, is still smaller than the higher degrees. Same observations are valid for the sparser settings. However, we should note that in sparser scenarios with a degree 2, we have observed unconnected nodes since the nodes could not find an available parent. Then, the minimum degree should be 3 for these scenarios since we aim to schedule connected topologies.

Figure 13 shows the scheduling performance when there is no degree constraint or the degree constraint is 3 with a single frequency and with multi-frequencies (16). On a single channel, we achieve some reduction in the number of

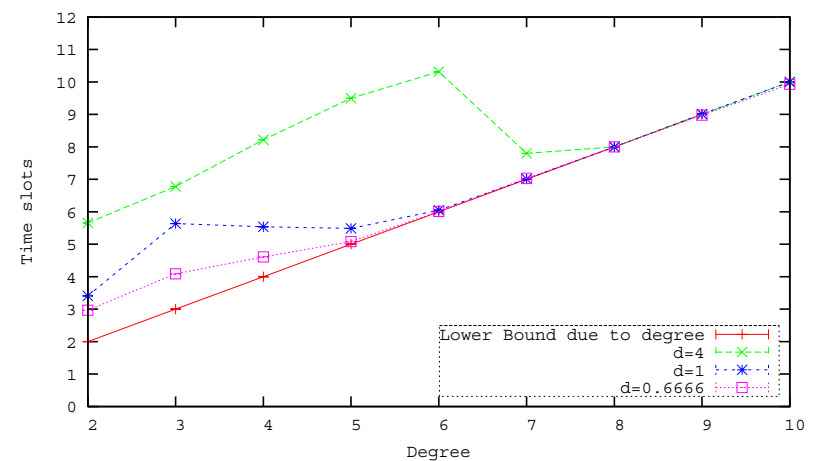

Fig. 12. Number of Time slots versus Maximum Routing Tree Degree

time slots compared with the baseline with non-constrained minimum-hop routing. However, due to the interference effects the number of required time slots is still high. On the other hand, minimum-hop routing on degree constrained trees combined with multi-frequency communication achieves a greater reduction on the schedule length. In denser deployments, for instance when $d=4$ the reduction is from 99 time slots to 7 time slots. In sparser deployments, for instance when $d=0.666\left(150 \times 150 \mathrm{~m}^{2}\right.$ area $)$, we achieve a reduction from 26 time slots to 4 time slots. In general, considering different densities, it can be concluded that an order of magnitude reduction in the number of time slots is achieved.

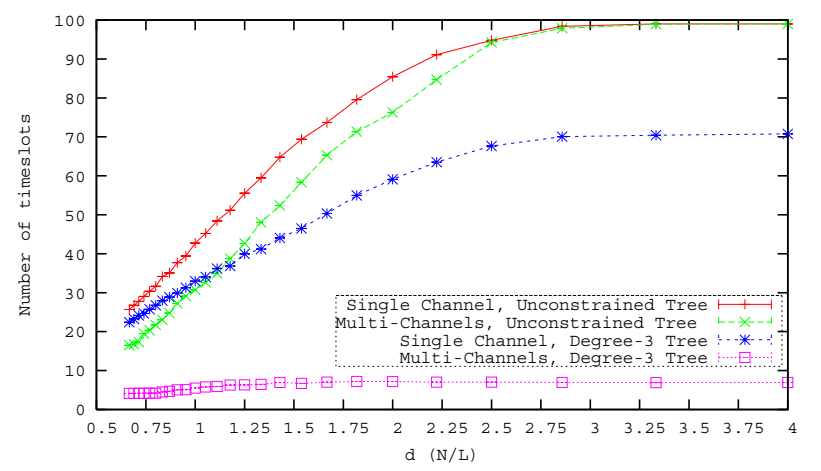

Fig. 13. Number of Time slots versus Density, Degree Constraint $=3$

\section{RELATED WORK}

A closely related study by Gandham et al. [13] focuses on finding a TDMA schedule that minimizes the total time required to complete a convergecast in WSN. The aim is to guarantee a bound on convergecast latency. They propose a scheduling algorithm that requires at most $3 N$ time slots where $N$ is the number of nodes in the network. Although, they address the same problem as ours, we focus on methods that can reduce the schedule length by eliminating the limitations due to interference and connectivity where they are interested in showing the bounds on the schedule length with different network organizations. Minimum frame length scheduling for TDMA wireless networks is also studied by Das et al. [14]. They develop mixed integer linear programming models for solving the problem optimally but their focus is only on power control and they consider the availability of sectored antennas. 
Joint scheduling and transmission power control is a well studied method [1], [5], [15], [16] to increase the throughput by limiting the interference effects in wireless networks. Moscibroda theoretically proves that non-linear power control mechanisms can significantly help to minimize the scheduling complexity and also improve the capacity of WSN even in the worst case [1]. However, we show that in a practical setting where the nodes have limited discrete transmission power levels it may not be always possible to overcome the excessive interference.

The use of multiple frequency channels is an efficient way to improve the capacity of wireless networks [17], [18], [19]. If the number of frequencies is sufficient and frequencies are assigned properly, the excessive levels of interference can be eliminated. Different than the previous work, we introduce a simple frequency and time scheduling method. Instead of assigning frequencies to the links or nodes we consider a receiver based frequency assignment which is suitable for aggregated data collection on a tree topology.

There exist studies evaluating the performance of graph based and SINR based interference models [6], [20]. Grönkvist et al. [6] report that the graph based models may result in serious interference since the model does not consider the accumulated interference. Our observations confirm the already reported results and we further give implications on which topologies the graph based models may not be accurate according to the density of the network and the path loss metric.

\section{CONCLUSIONS}

We have explored a number of techniques to enhance the aggregated data collection over a tree topology in WSN. Our initial approach was to use interference-aware minimum frame-length TDMA-scheduling that enables spatial reuse. The second step was to combine the scheduling with transmission power control. Although the well studied transmission power control method helped to overcome interference and reduce schedule length, it was found to be not always the best solution in a practical setting due to the limitations on the power settings of the nodes. The next step was to consider the use of multiple frequency channels. With the extensive simulations we found that for networks of about a hundred nodes, the use of multi-frequency scheduling can suffice to eliminate most of the interference. Then, data collection rate was no longer interference-limited, but rather topology-limited. Thus, our final approach was to use an appropriate degree-constrained tree construction. Simulation results showed that, combining the last two techniques can provide an order of magnitude improvement compared to the simple approach of scheduling on a single channel with minimum-hop routing trees.

We also evaluated the impact of different design choices to model interference. We concluded that graph based interference models may result in serious interference especially in sparser deployments. Furthermore, adjacent channel interference cannot always be ignored since the orthogonality of frequencies is dependent on the transceiver characteristics.

The gains with the degree constrained trees may be costly in terms of latency due to the increased number of hop distances to the sink node. As a future work, we are interested in addressing this open issue. Another issue to be considered is that some nodes may have a lot of data that require more than one time slot per frame while some others do not have any data to fill a time slot, hence the bandwidth may be wasted. That would be interesting to explore the scheduling performance in such scenarios.

\section{REFERENCES}

[1] T. Moscibroda, "The worst-case capacity of wireless sensor networks," in IPSN '07: Proceedings of the 6th international conference on Information processing in sensor networks, 2007, pp. 1-10.

[2] K. Chintalapudi, T. Fu, J. Paek, N. Kothari, S. Rangwala, J. Caffrey, R. Govindan, E. Johnson, and S. Masri, "Monitoring civil structures with a wireless sensor network," IEEE Internet Computing, vol. 10, no. 2, pp. 26-34, 2006.

[3] V. Rajendran, K. Obraczka, and J. J. Garcia-Luna-Aceves, "Energyefficient collision-free medium access control for wireless sensor networks," in Proceedings of ACM SenSys, 2003, pp. 181-192.

[4] J. Mao, Z. Wu, and X. Wu, "A tdma scheduling scheme for many-toone communications in wireless sensor networks," Comput. Commun., vol. 30, no. 4, pp. 863-872, 2007.

[5] T. A. ElBatt and A. Ephremides, "Joint scheduling and power control for wireless ad-hoc networks," in Proceedings of IEEE INFOCOM, vol. 2, Jun 2002, pp. 976-984.

[6] J. Grönkvist and A. Hansson, "Comparison between graph-based and interference-based stdma scheduling," in Proceedings of ACM MobiHoc, 2001, pp. 255-258.

[7] A. Mishra, V. Shrivastava, S. Banerjee, and W. Arbaugh, "Partially overlapped channels not considered harmful," SIGMETRICS Perform. Eval. Rev., vol. 34, no. 1, pp. 63-74, 2006.

[8] O. D. Incel, S. Dulman, P. Jansen, and S. Mullender, "Multi-channel interference measurements for wireless sensor networks," in Proceedings of LCN 2006, 2006, pp. 694-701.

[9] A. Gibbons, Algorithmic Graph Theory. Cambridge University Press, 1985.

[10] "Cc2420 single-chip $2.4 \mathrm{ghz}$ ieee 802.15 .4 compliant and zigbee $(\mathrm{tm})$ ready rf transceiver," 2007. [Online]. Available: http://www.ti.com/lit/gpn/cc2420

[11] "Nordic semi-conductors, nrf905 multiband transceiver," 2007. [Online]. Available: http://www.nordicsemi.com

[12] S. Khuller, K. Lee, and M. Shayman, "On degree constrained shortest paths," Lecture Notes in Computer Science, vol. 3669/2005, pp. 259$270,2005$.

[13] S. Gandham, Y. Zhang, and Q. Huang, "Distributed minimal time convergecast scheduling in wireless sensor networks," in ICDCS '06: Proceedings of the 26th IEEE International Conference on Distributed Computing Systems, 2006, pp. 50-57.

[14] A. K. Das, R. J. M. II, P. Arabshahi, and A. Gray, "Power controlled minimum frame length scheduling in tdma wireless networks with sectored antennas." in Proceedings of INFOCOM, 2005, pp. 1782-1793.

[15] R. L. Cruz and A. Santhanam, "Optimal routing, link scheduling, and power control in multi-hop wireless networks," in Proceedings of IEEE INFOCOM, 2003, pp. 702-711.

[16] R. Bhatia and M. Kodialam, "On power efficient communication over multi-hop wireless networks: joint routing, scheduling and power control," in Proceedings of IEEE INFOCOM, vol. 2, 2004, pp. 1457-1466.

[17] P. Bahl, R. Chandra, and J. Dunagan, "Ssch: slotted seeded channel hopping for capacity improvement in ieee 802.11 ad-hoc wireless networks," in Proceedings of ACM MobiCom, 2004, pp. 216-230.

[18] J. So and N. H. Vaidya, "Multi-channel mac for ad hoc networks: handling multi-channel hidden terminals using a single transceiver," in Proceedings of ACM MobiHoc, 2004, pp. 222-233.

[19] G. Zhou, C. Huang, T. Yan, T. He, J. Stankovic, and T. Abdelzaher, "Mmsn: Multi-frequency media access control for wireless sensor networks," in Proceedings of IEEE Infocom, 2006, pp. 1-13.

[20] A. Iyer, C. Rosenberg, and A. Karnik, "What is the right model for wireless channel interference?" in QShine '06: Proceedings of the $3 r d$ international conference on Quality of service in heterogeneous wired/wireless networks, 2006, pp. 2-11. 